\title{
CANSANCIO Y SOMNOLENCIA DURANTE EL DESEMPEÑO LABORAL DE LOS CONDUCTORES INTERPROVINCIALES: EXPERIENCIA PERUANA Y PLANTEAMIENTO DE PROPUESTAS
}

\author{
Jorge Rey de Castro 1,2,3,a,b Edmundo Rosales-Mayor 1,2,3,b,c
}

\begin{abstract}
RESUMEN
La información indica que los accidentes de tránsito causados por somnolencia o cansancio de los conductores de ómnibus son frecuentes en nuestro país. Un conductor que se duerme durante la conducción no puede realizar maniobras evasivas para evitar colisiones o despistes, siendo el resultado de este tipo de accidentes, gran número de víctimas y la destrucción de infraestructura. En este artículo se discute la información original publicada en Perú hasta la actualidad y plantea propuestas generales para enfrentar el problema.
\end{abstract}

Palabras clave: Accidentes de tránsito; Trastornos por excesiva somnolencia; Privación de sueño; Conducción de automóvil (fuente: DeCS BIREME).

\section{TIREDNESS AND SLEEPINESS IN RURAL BUS DRIVERS DURING THEIR JOB PERFORMANCE: PERUVIAN EXPERIENCE AND PROPOSALS}

\begin{abstract}
The information indicates that the traffic accidents caused by bus drivers' sleepiness or tiredness are frequent in our country. A driver that falls asleep while driving cannot perform evasive maneuvers in order to avoid crashes or getting off the track, being the result of this kind of accidents a great number of victims and infrastructure destruction. In this article we discuss the original data published in Peru up to date and make general proposals to face the problem.
\end{abstract}

Key words: Accidents, Traffic; Disorders of excessive somnolence; Sleep deprivation, Automobile driving (source: $\mathrm{MeSH}$ NLM).

\section{INTRODUCCIÓN}

Las publicaciones médicas extranjeras dan cuenta del cansancio y la somnolencia como causas de accidentes durante la conducción ${ }^{(1-5)}$. Los conductores fatigados o somnolientos disminuyen progresivamente el nivel de atención y concentración durante el manejo y pierden capacidad de respuesta ante condiciones que exigen reacciones inmediatas cuando circulan por la ciudad o las carreteras. Pestañear y dormitar durante la conducción reflejan un nivel extremo de fatiga y deuda de sueño. Estos accidentes tienen altísima siniestralidad en términos de muertos, heridos y pérdidas materiales. La información foránea señala que entre 4 a $30 \%$ de los accidentes en carreteras son producidos por el cansancio o somnolencia durante la conducción (1-2).
No existe prueba diagnóstica para documentar que la somnolencia o el cansancio del conductor son las causas de un accidente. Por ello, indagar por las características del siniestro permite concluir que la somnolencia estaría involucrada en su origen. Según el informe de la National Sleep Foundation (NSF) ${ }^{(6)}$ y el National Center for Sleep Disorders Research/ National Highway Traffic Safety Administration (NCSDR/NHTSA) ${ }^{(7)}$ las características que rodean al escenario son: el accidente ocurre durante la noche generalmente entre las 00.00-7.00 horas y en horario vespertino entre las 13.00-15.00 horas, el conductor maneja solo, la unidad sale inadvertidamente de la ruta o invade carril contrario y, el conductor no deja huellas de haber intentado una maniobra evasiva para evitar la colisión.

\footnotetext{
Centro de Trastornos Respiratorios del Sueño (CENTRES), Clínica Anglo Americana. Lima, Perú.

Facultad de Medicina, Universidad Peruana Cayetano Heredia. Lima, Perú.

Grupo de Investigación en Sueño (GIS). Lima, Perú.

Médico neumólogo; ' Magister en Medicina; Médico, Magister en Sueño.
}

Recibido: 19-02-10 Aprobado: 02-06-10 
De acuerdo a la información publicada por Planzer, la tasa de mortalidad en Perú debido a accidentes de tránsito fue 23 por 10 mil vehículos y ello nos ubicaría mundialmente en el cuarto lugar luego de Bangladesh, China y Sri Lanka (8). Si calculamos esta misma tasa a partir del ranking de las primeras 50 empresas de ómnibus involucradas en accidentes de tránsito registrados el año 2008 por el Ministerio de Transportes y Comunicaciones (MTC), la tasa es 2145 muertos por cada diez mil ómnibus circulantes, siendo éstas cifras notoriamente alarmantes (9). De otro lado, el Informe Defensorial 108 demuestra claramente la alta siniestralidad ocasionada por los accidentes de ómnibus interprovinciales, comparados con el total de accidentes registrados en la red nacional vial entre los años 2002 a 2005. De acuerdo con esta fuente, un accidente provocado por un ómnibus interprovincial causa 2,63 veces más heridos y 2,33 más muertos que el resto de las unidades ${ }^{(10)}$.

EI MTC registra en su portal electrónico ${ }^{(11)}$ las causas de los accidentes ocurridos en el país. El año 2008; exceso de velocidad, imprudencia del conductor, imprudencia del peatón y la categoría otros, ocasionaron accidentes en $30 \%, 26 \%, 9 \%$ y $18 \%$ de los casos, respectivamente (11). Si bien es cierto la información corresponde al universo de accidentes y no está limitado a los provocados por ómnibus interprovinciales ¿Cuáles son los criterios para calificar un accidente? Al parecer no hay una definición clara y uniforme de los términos por lo que imprudencia del conductor y otros podrían corresponder a un cajón de sastre que esconde otras causas no identificadas. Esta observación tiene mayor trascendencia en la medida que estas constituyeron el $44 \%$ de las causas de accidentes. Por lo tanto, y de acuerdo con las estadísticas mencionadas, los accidentes debido al cansancio o somnolencia del conductor no existirían en el Perú. Nuestras investigaciones demuestran lo contrario.

\section{DEFINICIÓN Y CAUSAS DE SOMNOLENCIA}

Una definición operacional de la somnolencia es la tendencia de la persona a quedarse dormido, también conocido como la propensión a dormirse o la habilidad de transición de la vigilia al sueño ${ }^{(12-14)}$. Las causas de la excesiva somnolencia puede ser primarias 0 secundarias (14-15). Dentro de las causas primarias, o también conocidas como de origen central, se encuentran la narcolepsia, la hipersomnia idiopática y otras hipersomnias primarias poco frecuentes como el síndrome Kleine-Levin.

Dentro de las causas secundarias podemos mencionar dos grandes grupos. El primero serían los trastornos que ocurren durante o relacionados con el sueño como por ejemplo, los trastornos respiratorios siendo el más conocido el síndrome de apnea - hipopnea del sueño, comportamientos que lleven a privación del sueño como trabajar de noche u otros trastornos del sueño como el jet lag o cualquier otra alteración del ciclo circadiano, el síndrome de piernas inquietas o el movimiento periódico de miembros. $Y$ el segundo grupo que engloba a diversas condiciones médicas como traumas encefálicos, accidentes cerebro-vasculares, cáncer, enfermedades inflamatorias, encefalitis o condiciones neurodegenerativas o psiquiátricas, especialmente la depresión; asimismo, se incluye los efectos de ciertos medicamentos como las benzodiacepinas.

\section{LO ESTUDIADO EN PERÚ}

La primera aproximación al tema tuvo su origen en la simple observación de los artículos periodísticos publicados en los diarios del país. Se trataba de accidentes de ómnibus de pasajeros en que las circunstancias que rodeaban al siniestro sugerían que el cansancio o la somnolencia del conductor podrían ser responsables del accidente o que en el texto del artículo, el periodista atribuía el accidente a dicha causa. El año 2001 implementamos una revisión de la base de datos del diario El Comercio, registrándose todos los accidentes de ómnibus ocurridos en las carreteras del país entre 1999-2000. Los artículos fueron clasificados como tipo A si el periodista afirmaba en el titular o texto del artículo que el conductor se quedó dormido o que se habría quedado dormido. Tipo B si el artículo periodístico describía al menos dos criterios altamente sugestivos de accidente causados por somnolencia o cansancio. Los criterios fueron: accidentes ocurridos entre las 01.00-06.00 horas o 13.00-15.00 horas, sin evidencia de maniobra evasiva, colisión frontal con otro vehículo, unidad invadió carril contrario, cayó a un barranco o colisionó con una edificación, conductor viajó sólo y jornada previa al accidente mayor de ocho horas. En el periodo mencionado, el diario El Comercio publicó 73 artículos dando cuenta de 112 accidentes; doce (11\%) fueron de tipo A y 41 (37\%) tipo B. Prácticamente la mitad de los siniestros desde la perspectiva periodística tuvieron alguna relación con el cansancio o somnolencia del conductor. Las unidades directamente involucradas fueron ómnibus 38 (34\%) y camiones 31 (27\%). Con todas las limitaciones que implica la sistematización de la información referida, estos resultados sugerían la existencia de accidentes en nuestras carreteras debido a la somnolencia o cansancio del conductor ${ }^{(16)}$.

El año 2001 implementamos un estudio cualitativo diseñado con la finalidad de conocer el estilo de vida de los conductores de ómnibus de pasajeros y la relación entre 
somnolencia y accidentes de tránsito en la carretera Panamericana Norte. Empleamos la metodología cualitativa con tres grupos focales. Los conductores participantes laboraban en el terminal terrestre informal más grande de la República Peruana, ubicado en la zona norte de Lima, el Terminal de Fiori. La información obtenida indicó que los conductores realizaban jornadas prolongadas sin programación sistemática que permitiera un adecuado descanso, se alternaban desordenadamente turnos diurnos y nocturnos descansando un promedio de cuatro a cinco horas por día. Los conductores, luego de llegar al terminal terrestre, debían dedicarse a la limpieza de la unidad lo que prolongaba sus horas de labor y reducía el descanso. Empleaban una serie de maniobras para evitar quedarse dormidos; como: fumar, beber café, escuchar música, abrir la ventana para refrescarse, comer frutas, mojarse la cara, beber mezclas de alcohol con hoja de coca y mezclas de gaseosas a base de cafeína con café. Los conductores relataron eventos que reflejaban compromiso neurocognitivo durante la conducción como: desorientación geográfica, pérdida de memoria reciente y compromiso del juicio. Destacaron también compromiso de tipo motor asociado claramente al cansancio como dificultades para el cambio de marchas. Los conductores describieron eventos de microsueños que fueron expresamente identificados como espejismos, particularmente frecuentes en las horas de madrugada así como relatos de pestañeo durante la conducción. Todos los conductores que participaron en los grupos focales reconocieron que alguna vez se habían quedado dormidos durante la conducción y cuatro tuvieron experiencias de haber estado a punto de accidentarse ${ }^{(17-18)}$.

El mismo estudio permitió desentrañar condiciones de orden laboral deplorables. No hay sistematización ordenada en la programación de salida de los buses y muchas veces ello está sujeto al llenado de las plazas de pasajeros. No hay orden ni alternancia previamente establecida para los turnos día/noche. Se alternan dos conductores a lo largo de toda la ruta aproximadamente cada cuatro horas. El que descansa lo hace habitualmente en el maletero o portaequipaje del ómnibus. Este peligroso hábito se repite tanto cuando el vehículo está detenido en el terminal principal como durante su recorrido en ruta. Desafortunadamente el entorno informal es la regla más que la excepción lo que agrava el problema de forma considerable. EI terminal y paraderos principales no disponen de áreas de descanso que garanticen comodidad, silencio y oscuridad requeridas para un buen dormir. Ningún conductor tenía beneficios sociales reconocidos como seguro médico y vacaciones. Finalmente, los conductores visitaban a sus familias entre cada 7 a 16 días ${ }^{(17-18)}$.

En marzo de 2002 fue realizado un estudio transversal, prospectivo, descriptivo y no probabilístico en conductores de la misma terminal. Se encuestaron 238 conductores de una población total de 400 . Se aplicó un cuestionario supervisado con 23 preguntas cuyo diseño fue construido a partir de las herramientas de McCartt et al. ${ }^{(19)}$, National Sleep Foundation ${ }^{(20-21)}$ y un cuestionario de uso clínico ${ }^{(22)}$. El $47 \%$ de los encuestados dormían menos de siete horas diarias y $40 \%$ menos de seis. Al momento de la encuesta, el $31 \%$ de los conductores habían dormido menos de seis horas de las últimas veinticuatro. Hasta $80 \%$ de los participantes reconocieron que solían conducir más de cinco horas sin detenerse. El 56\% había presentado cansancio durante la conducción desde "alguna vez" hasta "siempre". La sensación de cansancio fue más marcada en la madrugada en el $65 \%$ y durante las tardes en $20 \%$ de los participantes. La tercera parte reconoció haber pestañeado y sentido cansancio durante la conducción.

El estudio cuantitativo confirmó las maniobras utilizadas por los conductores para evitar quedarse dormidos durante su trabajo. La costumbre de dormir en el maletero fue confirmada en el $81 \%$ de los conductores, la mitad de los cuales lo hacía en el terminal o cuando alternaba con el segundo conductor durante el recorrido de la ruta. El $45 \%$ de los participantes admitieron haber tenido situaciones de casi accidente o haberse accidentado durante la conducción. Este informe documentó, por primera vez en el Perú, que los casi accidentes o accidentes reconocidos por los conductores entrevistados sucedieron preferentemente entre las 02.00 y 06.00 horas de la madrugada ${ }^{(23)}$ (Figura 1). El análisis bivariado reveló relación estadísticamente significativa entre pestañeo y cansancio del conductor y la variable haber estado a punto de accidentarse o haber tenido un accidente. Los conductores opinaron que la principal causa de accidentes en el país fue el cansancio.

En julio de 2007 Rosales E. et al. desarrollaron una encuesta similar en el Terminal Terrestre de Huancayo, provincia de Huancayo en la región de Junín en la sierra central de Perú ${ }^{(24)}$, en la que participaron 100 conductores de una población total aproximada de 200. Los encuestados dormían en promedio 6,8 horas los días que trabajaban y 9,9 horas los días que no lo hacían. Las 24 horas previas a la encuesta $47 \%$ había dormido seis horas o menos y $26 \%$ conducía más de cuatro horas sin detenerse. El 98\% de los participantes reconocieron que conducían de noche y tres de cada cuatro lo hacían cinco o más noches por semana. El $32 \%$ había presentado cansancio durante conducción y ello se había presentado principalmente durante la noche y la madrugada. El $17 \%$ había pestañeado y el $4 \%$ cabeceó durante la conducción.

Como fuera descrito en el estudio del Terminal Fiori, el $65 \%$ de los conductores tiene la costumbre de dormir 
en el maletero del ómnibus. El 59\% reconoció haber tenido algún accidente o situación de casi accidente. El $40 \%$ de los conductores que tuvieron esta experiencia, mencionó que la causa principal fue el cansancio. La hora aproximada del accidente o casi accidente fue muy similar al estudio realizado en el Terminal de Fiori, entre las 01.00 y 04.00 horas (Figura 1). En el análisis bivariado y multivariado, nuevamente se encontró que existía asociación significativa entre el cansancio, pestañeo y cabeceo durante la conducción con haberse accidentado o haber estado a punto de accidentarse. La mayoría de los encuestados (82\%) opinaron que la causa principal de los accidentes en las carreteras del Perú era el cansancio.

El año 2008, Liendo et al., desarrollaron un estudio en conductores de ómnibus interprovinciales con la finalidad de evaluar las diferencias entre empresas registradas en el MTC como formales frente a informales ${ }^{25)}$. Los autores definieron a priori criterios arbitrarios para calificar al conductor encuestado como formal o informal si cumplían con cinco normas pautadas en dos decretos supremos ${ }^{(26-27)}$. De acuerdo con estos criterios el $11 \%$ de los conductores formales dormía menos de cinco horas al día, $63 \%$ trabajaba cuatro y más noches por semana, y las horas en la que se producían más eventos de accidentes o casi accidentes fue entre la 00.00 y 04.00 horas de la madrugada (Figura 1). Ninguna de estas variables tuvo diferencia estadísticamente significativa al ser comparadas con conductores informales (Figura 1).

La información recopilada indica, contrariamente a los datos oficiales, que una proporción no precisada de ac- cidentes de ómnibus en carreteras son causados por la somnolencia o cansancio del conductor. Las condiciones laborales de los conductores dejan mucho que desear, los horarios de trabajo no están debidamente sistematizados, no hay ambientes adecuados para el descanso lo que lleva a mala calidad, privación aguda y crónica del sueño. Los conductores de ómnibus interprovinciales trabajan cansados y somnolientos poniendo en peligro la vida y salud de sus pasajeros sin que medie intervención alguna de las autoridades para controlar el riesgo.

Desafortunadamente, las sucesivas normativas planteadas por el MTC son letra muerta ${ }^{(26-27)}$ y el escenario de la informalidad agrava tremendamente el problema. Es irresponsable y absolutamente fuera de sustento atribuir exclusivamente a los conductores la culpabilidad de los accidentes en las carreteras. La responsabilidad primaria pasa por todas las autoridades gubernamentales desde el ámbito legislativo, ejecutivo, judicial, control tributario y propietarios de las empresas de transporte. El Estado no debe dejar la iniciativa al caos, desorden e informalidad sino por el contrario ocupar el espacio y funciones que le corresponde.

\section{PROPUESTAS PARA DISMINUIR LA TASA DE ACCIDENTES EN LAS CARRETERAS DEL PERÚ}

Basados en la información existente hasta la actualidad tanto en el contexto local como internacional, planteamos distintas propuestas que pueden ser aplicadas a diferentes niveles (Tabla 1)

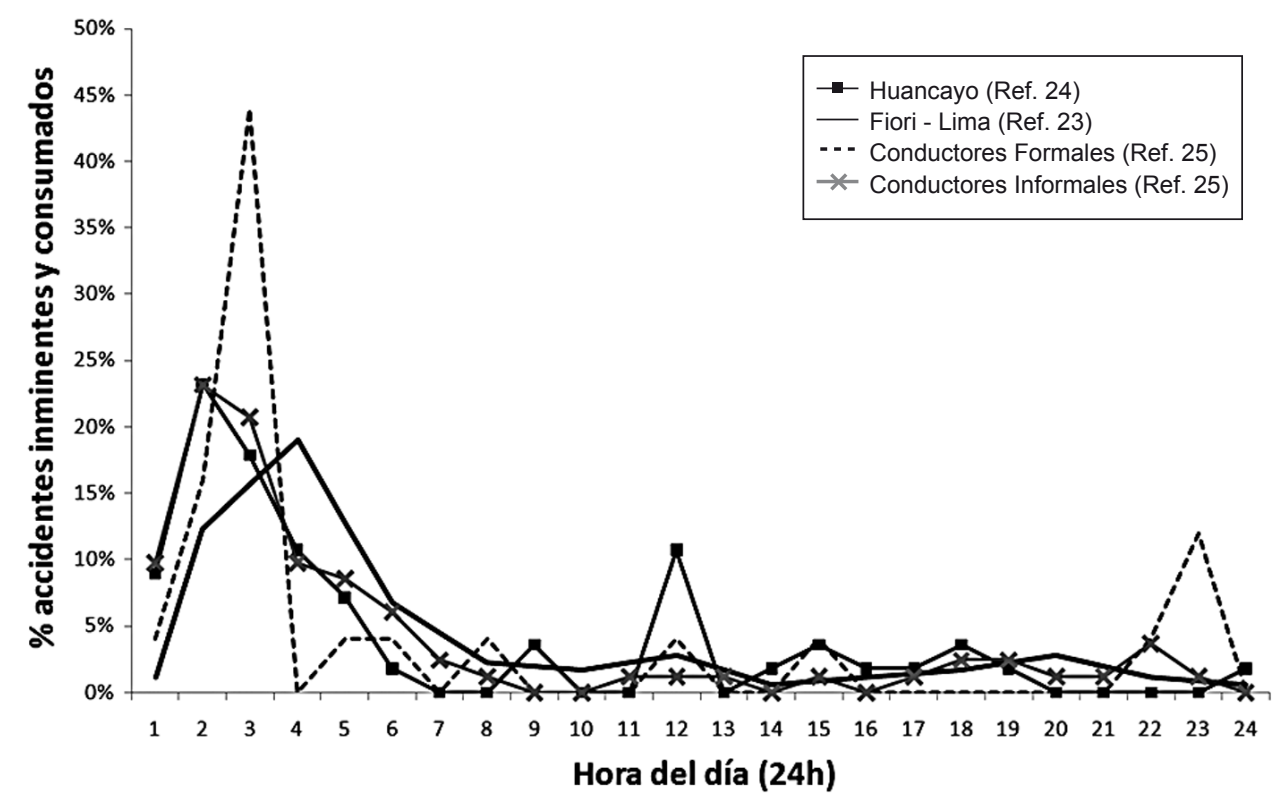

Figura 1. Hora en la que sucedieron los accidentes o casi accidentes de tránsito reportados por los conductores. 


\section{A NIVEL DE INDIVIDUOS}

Es necesario diseñar intervenciones educativas con la finalidad de promover buenos hábitos de alternancia sueño-vigilia entre conductores, y hacerlos extensivos a los propietarios de las empresas de transportes ${ }^{(5-7)}$.

\section{A NIVEL LABORAL}

Los turnos de trabajo de los conductores deben sistematizarse ordenadamente. No es recomendable conducir más de cuatro horas continuas ni debe propugnarse más de dos turnos de cuatro horas, cada uno en un periodo de 24 horas, en otras palabras, un conductor puede conducir hasta un máximo de ocho horas al día con un descanso de cuatro horas de por medio.

La conducción durante la madrugada debe restringirse progresivamente y para el caso de los conductores que trabajan en turnos nocturnos deben hacerlo por un máximo de cuatro horas en toda la noche, promoviéndose

Tabla 1. Resumen de las propuestas para disminuir la tasa de accidentes de tránsito debidos a la somnolencia o cansancio del conductor de ómnibus.

\section{A nivel de individuos}

- Promover buenos hábitos de sueño.

\section{A nivel laboral}

- No conducir más de cuatro horas continuas, y hasta un máximo de ocho horas en un período de 24 horas.

- Restringir la conducción durante la madrugada.

- Promover que los viajes se realicen en turno diurno.

- Acondicionar habitaciones en las terminales que sirvan para el descanso de los conductores. No deben usarse bajo ninguna circunstancia las bodegas o maleteros.

- El conductor no debe asumir la labor de la limpieza o mantenimiento de la unidad de transporte.

- Proporcionar a los conductores las prestaciones sociales determinadas por ley dentro del contexto laboral formal.

\section{A nivel de instituciones}

- Formalización del sector transporte.

- Incluir dentro de las estadísticas a la somnolencia o cansancio del conductor como causa de accidente como una categoría individual.

\section{A nivel de la comunidad científica}

- Extender la investigación sobre la somnolencia como causa de accidentes de tránsito a conductores de otros. tipos de transporte, sean estos particulares o públicos. entre propietarios de ómnibus y entre pasajeros el uso de turnos diurnos para movilizarse entre los diferentes destinos del país y disminuir los viajes en turno nocturno que incluyan especialmente las madrugadas, ya que ello disminuiría la exposición al riesgo de accidentes durante las horas en las que el conductor tiende a estar más somnoliento y cansado ${ }^{(5-7)}$.

Bajo ninguna circunstancia los conductores deben dormir en las bodegas o maleteros y, por el contrario, las empresas de transportes deben acondicionar habitaciones en los terminales y a lo largo de la ruta, donde el conductor pueda dirigirse a descansar de inmediato una vez finalizado su recorrido. El conductor no debe asumir la responsabilidad de la limpieza o mantenimiento mecánico de la unidad una vez que arriba a su destino. Insistir con esta costumbre sólo reduce el periodo de descanso y sueño correspondiente.

Dentro de un contexto laboral formal, se debe proporcionar a los conductores las prestaciones sociales que les corresponden de acuerdo a ley como son: seguro social, descansos programados, vacaciones, gratificaciones y jubilación.

\section{A NIVEL DE INSTITUCIONES}

La formalización de todas las empresas de transporte terrestre es imperativa y prioritaria, ello permitirá un adecuado control y aplicación de normas bajo la responsabilidad de las autoridades y el Estado en su conjunto que apunten a disminuir la alta tasa de siniestralidad en las carreteras de la República.

¿Por qué la somnolencia no aparece en las estadísticas oficiales del MTC?, es una pregunta que no podemos responder pero una indagación seria y sistematizada de la metodología empleada para la calificación de los accidentes debe ser motivo de investigaciones futuras. Debe dársele más importancia al trabajo de los peritos de la Policía Nacional del Perú (PNP) y evitar que su participación se limite exclusivamente a accidentes que involucran muertes. La intervención del perito debe incluir un ítem sistematizado para calificar somnolencia o cansancio del conductor como causa probable del accidente.

\section{A NIVEL DE LA COMUNIDAD CIENTÍFICA}

Recomendamos incentivar la investigación en el tema que debe ser extendida a conductores de ómnibus de pasajeros y camiones en el resto de la república. También deberá indagarse en conductores de microbuses, combis, taxis y automóviles particulares en las zonas urbanas. 


\section{CONCLUSIÓN}

Promover el adecuado descanso de los choferes de ómnibus protegerá a los pasajeros del riesgo de morir o presentar secuelas motoras o neurológicas de por vida. Apuntemos a un objetivo concreto a partir de los hallazgos descritos: un conductor alerta y descansado garantiza más seguridad a los usuarios.

\section{Fuente de Financiamiento}

Autofinanciado.

\section{Conflictos de Interés}

Los autores declaran no tener conflicto de interés en la publicación de este artículo.

\section{REFERENCIAS BIBLIOGRÁFICAS}

1. Horne JA, Reyner LA. Sleep related vehicle accidents. BMJ. 1995; 310(6979): 565-7.

2. Sagberg F. Road accidents caused by drivers falling asleep. Accid Anal Prev. 1999; 31(6): 639-49.

3. Mitler MM, Miller JC, Lipsitz JJ, Walsh JK, Wylie CD. The sleep of long-haul truck drivers. N Engl J Med. 1997; 337(11): 755-61.

4. Lyznicki JM, Doege TC, Davis RM, Williams MA. Sleepiness, driving, and motor vehicle crashes. Council on Scientific Affairs, American Medical Association. JAMA. 1998; 279(23): 1908-13.

5. Dement WC, Mitler MM. It's time to wake up to the importance of sleep disorders. JAMA. 1993; 269(12): 1548-50.

6. National Sleep Foundation. Drowsy driving: detection and prevention. [Página en Internet] Washington DC, Estados Unidos de Norteamérica; 2010 [Actualizado 2010; Fecha de acceso: 01 de abril del 2010]. Disponible en: http:// drowsydriving.org/about/detection-and-prevention

7. National Center for Sleep Disorders Research and National Highway Traffic Safety Adminstration Expert Panel on Driver Fatigue and Sleepiness. Drowsy driving and automobile crashes: report and recommendations. Washington DC: National Heart, Lung, and Blood Institute National Center for Sleep Disorders Research; 1998.

8. Planzer R. La seguridad vial en la región de América Latina y el Caribe. Situación actual y desafíos. Santiago de Chile: Naciones Unidas - Comisión Económica para América Latina y el Caribe (CEPAL); 2006.

9. Ministerio de Transportes y Comunicaciones. Ranking de empresas de transporte interprovincial de personas de ámbito nacional con el más alto índice de participación en accidentes de tránsito. Lima: Ministerio de Transportes y Comunicaciones; 2008.

10. Defensoría del Pueblo. Informe Defensorial № 108. Pasajeros en riesgo: la seguridad en el transporte interprovincial. Lima: Defensoría del Pueblo; 2008.

11. Ministerio de transportes y Comunicaciones. Perú: Número de accidentes de tránsito fatales y no fatales por año según causa 2000-2008. Lima, Ministerio de Transportes y Telecomunicaciones; 2009.
12. Roehrs T, Carskadon MA, Dement WC, Roth T. Daytime sleepiness and alertness. In: Kryger MH, Roth T, Dement WC, Editores. Principles and practice of sleep medicine. 4ta ed. Philadelphia: Saunders; 2005. p. 39-49.

13. Silber $\mathbf{M}$. The investigation of sleepiness. Sleep Med Clin. 2006;1: 1-7.

14. Cluydts R, De Valck E, Verstraeten E, Theys $P$. Daytime sleepiness and its evaluation. Sleep Med Rev. 2002; 6(2): 83-96.

15. Pagel JF. Excessive daytime sleepiness. Am Fam Physician. 2009;79(5):391-6.

16. Rey de Castro J. Accidentes de tránsito en carreteras e hipersomnia durante la conducción. ¿Es frecuente en nuestro medio? La evidencia periodística. Rev Med Hered. 2003; 14(2): 69-73.

17. Rey de Castro J, Soriano S. Hipersomnia durante la conducción de vehículos ¿causa de accidentes en carreteras? A propósito de un estudio cualitativo. Rev Soc Peru Med Interna. 2002; 15(3): 142-49.

18. Soriano S, Rey de Castro J. Una aproximación a los choferes de omnibuses interprovinciales. Accidentes de tránsito y privación crónica del sueño en la carretera Panamericana. Antropológica. 2002 20(1): 231-46.

19. McCartt AT, Rohrbaugh JW, Hammer MC, Fuller SZ. Factors associated with falling asleep at the wheel among long-distance truck drivers. Accid Anal Prev. 2000;32(4):493-504.

20. Johnson EO. Sleep in America: 1999. Results from the Nacional Sleep Foundations 1999 Omnibus Sleep Poll. Washington DC: National Sleep Foundation; 1999.

21. National Sleep Foundation. 2002 "Sleep in America" poll: adult sleep habits. Washington DC: National Sleep Foundation; 2002.

22. Rey de Castro J, Vizcarra D. Síndrome apnea del sueño obstructiva: descripción clínica y polisomnográfica en 48 pacientes. Rev Soc Peru Med Interna. 1999;12(1):2-7.

23. Rey de Castro J, Gallo J, Loureiro H. Cansancio y somnolencia en conductores de ómnibus y accidentes de tránsito en el Perú: estudio cuantitativo. Rev Panam Salud Publica. 2004;16(1):11-8.

24. Rosales E, Egoavil M, Durand I, Montes N, Flores R, Rivera S, et al. Accidentes de carretera y su relación con cansancio y somnolencia en conductores de ómnibus. Rev Med Hered. 2009;20(2):136-47.

25. Liendo G, Castro C. Cansancio y somnolencia en conductores de ómnibus interprovinciales con base en Lima, según condiciones laborales de la empresa de transportes [Tesis de Bachiller]. Lima: Facultad de Medicina, Universidad Peruana Cayetano Heredia; 2009.

26. Perú, Ministerio de Transportes y Comunicaciones. Reglamento Nacional de Tránsito. Decreto Supremo 0332001-MTC de 24 de julio del 2001. Lima: MTC; 2001.

27. Perú, Ministerio de Transportes y Comunicaciones. Reglamento Nacional de Vehículo. Decreto Supremo 0582003-MTC de 07 de octubre del 2003. Lima: MTC; 2003.

Correspondencia: Jorge Rey de Castro

Correo electrónico: jorgerey@rcp.net.pe 